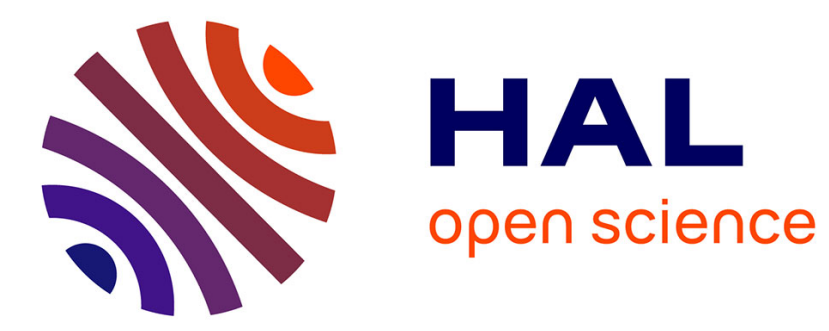

\title{
Quand les TIC réussissent trop bien dans les organisations : le cas du courrier électronique chez les managers \\ Sébastien Tran
}

\section{- To cite this version:}

Sébastien Tran. Quand les TIC réussissent trop bien dans les organisations: le cas du courrier électronique chez les managers. Revue management \& avenir, 2010, 34, pp.200-215. halshs-00638824

\section{HAL Id: halshs-00638824 \\ https://shs.hal.science/halshs-00638824}

Submitted on 6 Mar 2012

HAL is a multi-disciplinary open access archive for the deposit and dissemination of scientific research documents, whether they are published or not. The documents may come from teaching and research institutions in France or abroad, or from public or private research centers.
L'archive ouverte pluridisciplinaire HAL, est destinée au dépôt et à la diffusion de documents scientifiques de niveau recherche, publiés ou non, émanant des établissements d'enseignement et de recherche français ou étrangers, des laboratoires publics ou privés. 


\title{
Quand les TIC réussissent trop bien dans les organisa- tions : le cas du courrier électronique chez les managers
}

\author{
Sébastien TRAN \\ Professeur associé, Groupe Sup de Co La Rochelle-CEREGE \\ Chercheur associé à M-Lab (DRM - Université Paris Dauphine) \\ 102, rue de Coureilles \\ 17000 La Rochelle \\ Tel : 0546517700 - Fax : 0546517798 \\ Courriel : trans@esc-larochelle.fr
}

\section{Résumé}

Les managers dans la plupart des entreprises doivent faire face à une masse croissante d'information à traiter, ainsi qu'à des problématiques de coordination de plus en plus complexes dans les organisations du fait de la multiplication des unités de temps et de lieux. Parmi les outils mis à disposition des managers, le courrier électronique est l'une des premières technologies utilisées et les plus diffusées dans les entreprises. Ce medium apparait dès lors comme un objet de recherche approprié pour étudier la problématique d'articulation des TIC. Les récents travaux tels que la théorie du millefeuille ont souligné cette superposition des outils de communication dans les organisations sans une réelle logique de cohérence et de complémentarité, amenant alors une augmentation des canaux de communication. Nous chercherons à démontrer que les propriétés émergentes du médium et les usages des utilisateurs constituent une explication quant à la difficulté d'articuler plusieurs technologies censées être complémentaires et que le courrier électronique est devenu un point nodal structurant dans le portefeuille technologique des managers.

\section{Mots clefs :}

TIC, courrier électronique, théorie du millefeuille.

\section{Abstract}

In most companies managers have to cope with an ever increasing mass of information to deal with, along with coordination problems that are also more and more complex due to the multiplicity of time units and places. Among the tools managers have to hand is emailing, which is the most commonly used and widespread means of communication within companies. Therefore, this medium stands out as an appropriate matter for research in order to study how ICT interrelate. Recent studies such as the millefeuille theory have highlighted this stacking up of communication tools in corporations by which they do not complement each other nor show any coherence - and this led to a large number of communication channels. The purpose of this paper is firstly to bring out evidence that emailing, with its emerging idiosyncrasies, and how it is used by everyone actually explain why interrelating several technologies that are expected to complement each other is so dif- 
ficult - and secondly, to show how e-mailing had become indispensable and a prerequisite in managers' array of communication tools.

\section{Keywords :}

ICT, email, millefeuille theory. 


\section{Introduction}

Les managers dans les entreprises doivent faire face à une masse croissante d'information à traiter, ainsi qu'à des problématiques de coordination de plus en plus complexes dans les organisations du fait de la multiplication des unités de temps et de lieux, notamment dans les pays industrialisés. Face à cette problématique, les entreprises ont cherché à s'équiper avec les Technologies de l'Information et de la Communication (TIC) qui permettent de saisir, traiter, stocker et communiquer l'information (Reix, 2002). Leur mise en place et leur utilisation au fil du temps sont associées à l'application de processus de changement organisationnel (Reix, 1990 ; Marciniak et Rowe, 1997) qui sont très difficiles à cerner. La diffusion et l'appropriation des TIC dans les entreprises ne sont pas sans poser un certain nombre de questions, notamment dans le choix et l'articulation des différentes technologies censées aider les managers dans leurs activités quotidiennes. Les récents travaux tels que la théorie du millefeuille (Kalika et al., 2007) ont souligné cette superposition des outils de communication dans les organisations sans une réelle logique de cohérence et de complémentarité, amenant alors une augmentation des canaux de communication et une substitution entre les différents outils.

Parmi les outils mis à disposition des managers, le courrier électronique est l'une des premières technologies utilisées et les plus diffusées dans les entreprises. Au fil des années, l'email est ainsi devenu un moyen de communication incontournable dans l'entreprise (Rice et Gattiker, 2001 ; Katz et Rice, 2002 ; Van den Hooff et al, 2005) bien que son utilisation est évoluée au fur et à mesure de l'appropriation par les acteurs. Toutefois, même s'il existe plusieurs travaux antérieurs, les résultats demeurent contradictoires (Limayen et al., 1997 ; Raymond, 2002) et un certain nombre de questions restent en suspens et interrogent encore la communauté en Système d'Information (SI) (Weber, 2004). Enfin, il n'existe pas de définition qui fasse réellement consensus. Nous prenons donc la liberté de définir cet outil comme «un medium de communication asynchrone entre deux ou plusieurs personnes ou organisations basé sur des réseaux électroniques ouverts (Internet) et/ou privés permettant d'échanger des messages en format texte et des documents en format électronique $»$.

Ce medium apparaît dès lors comme un objet de recherche approprié pour étudier la problématique d'évolution et d'articulation des TIC. Nous chercherons à démontrer que les propriétés émergentes du médium et l'évolution des usages des utilisateurs constituent une explication quant à la difficulté d'articuler plusieurs technologies censées être pourtant complémentaires. Dans une première partie, nous présenterons un état des lieux de la diffusion du courrier électronique dans les entreprises en France et une revue de littérature sur l'évolution de cet outil dans les organisations. Ensuite, nous exposerons notre méthodologie centrée sur une approche qualitative à partir de plusieurs entretiens semi-directifs et nous dégagerons quelques éléments explicatifs pouvant offrir une base de réflexion quant aux problématiques d'articulation du courrier électronique avec d'autres TIC présentes dans les organisations. 


\section{Le courrier électronique : une TIC très diffusée et étudiée dans la lit- térature}

Le courrier électronique s'est imposé au fil des années comme un outil de communication incontournable dans l'organisation, dont les effets sont significatifs (Karahanna et Limayen, 2000) mais malgré tout très difficiles à déterminer. Le courrier électronique est marqué par des logiques sociales et organisationnelles qui expliquent sa large diffusion dans les entreprises, notamment dans les pays développés. Ainsi, plusieurs facteurs apparaissent dans la littérature pour expliquer sa diffusion : des facteurs contingents, des facteurs de situation, des facteurs liés à l'influence sociale (Boukef et Kalika, 2006). La généralisation du courrier électronique au sein des organisations témoigne d'une indéniable réussite de diffusion d'une technologie. Toutefois, elle soulève certaines questions à mesure de sa diffusion et des innovations technologiques incrémentales depuis plusieurs années.

\subsection{Le courrier électronique comme objet de recherche emblématique des TIC ?}

Le courrier électronique est l'une des premières technologies de communication à s'être largement diffusée au sein des entreprises en France, et ce aussi bien dans les PME que dans les grandes entreprises (SESSI, 2006, 2007). La complexité croissante dans les organisations en termes de coordination ou d'incertitude (Daft et Lengel, 1986; Galbraith, 1973) ont conduit ces dernières à mettre en place des outils de communication comme le courrier électronique. S'il est clair que la nécessité de communiquer et d'échanger des informations au sein et entre les organisations est une des explications de la pénétration de la messagerie électronique, cette dernière s'avère néanmoins insuffisante pour comprendre le niveau très important de diffusion et surtout d'appropriation de cet outil par les utilisateurs.

Le courrier électronique étant un outil de communication entre plusieurs individus et/ou organisations, sa diffusion se trouve être corrélée au développement d'une masse critique d'utilisateurs (Markus, 1987 ; 1990) et à des externalités de réseau positives (Katz et Shapiro, 1985). L'accès à Internet ${ }^{1}$ et l'équipement en micro-ordinateurs ont permis une diffusion et une utilisation croissante d'un outil comme le courrier électronique. Toutefois, l'accessibilité au courrier électronique n'engendre pas systématiquement un fort degré d'utilisation ${ }^{2}$, d'où la prudence dont il faut faire preuve lors de l'analyse et de l'interprétation de certains chiffres. Il n'existe pas de fracture numérique au sein des entreprises (entre les utilisateurs et les non-utilisateurs) mais plutôt une fracture des usages entre différentes manières d'utiliser les TIC et leur implication selon la nature des tâches à réali-

\footnotetext{
${ }^{1}$ En 2007, $92 \%$ des entreprises de 10 à 19 salariés sont connectées à Internet, contre $77 \%$ en 2003. Par ailleurs, $86 \%$ des entreprises d'au moins 10 salariés connectées disposent de l'ADSL, et $26 \%$ d'une connexion Internet d'au moins 2 Mbits/s (SESSI, 2007).

${ }^{2}$ Une étude du SESSI (2006) sur les entreprises industrielles montre que dans seulement $15 \%$ d'entre elles, plus de la moitié des salariés utilisaient le courrier électronique.
} 
$\mathrm{ser}^{3}$. D'autre part, l'hypothèse déterministe sous-jacente à de nombreuses réflexions est que plus on utilise la technologie, plus cela s'avère bénéfique pour l'organisation (productivité, coordination, diffusion des informations...) sans que cela ne puisse être réellement démontré dans les études ou travaux de recherche.

La réussite d'un SI (ou d'une de ses composantes) est évaluée le plus souvent dans les modèles conceptuels par plusieurs variables : le niveau d'utilisation de l'outil en question, sa facilité d'utilisation et la satisfaction des utilisateurs (Bailey et Pearson, 1983 ; Davis, 1989 ; Fulk et al. 1990 ; Delone et McLean, 2003). Si l'on s'en tient à la première variable, il est clair que même s'il persiste des différences sectorielles entre les différents types d'entreprise, le courrier électronique est l'une des TIC les plus diffusées dans les entreprises. Certains travaux soulignent que l'expérience d'utilisation du courrier électronique ou le nombre d'années d'expérience d'utilisation de l'outil sont positivement corrélés avec la fréquence de son utilisation (Linlin, 1996 ; Limayem, Bergeron et Richard, 1997). Le facteur de facilité d'utilisation est également utilisé dans la littérature sur l'acceptation d'une technologie par les utilisateurs et leurs comportements vis-à-vis de cette dernière. Le courrier électronique est un média simple, relativement intuitif et diffusé par ailleurs aussi bien dans le domaine public que dans le domaine professionnel, ce qui facilite l'effet d'apprentissage. L'ergonomie de l'outil a joué un rôle déterminant dans le niveau et le temps d'adoption de la technologie ${ }^{4}$.

\subsection{Le courrier électronique : un outil dépassant une logique communicationnelle ?}

Parmi les différentes approches possibles de la technologie, celle du paradigme sociotechnique nous paraît la plus pertinente par rapport à notre problématique. Le point de départ de cette approche est de considérer l'organisation comme un construit social structuré par des acteurs (individuels ou collectifs) et par des systèmes de relations et de communication. Les travaux de Crozier et Friedberg (1977) nous permettent de considérer l'organisation comme une forme repérable où ont lieu des actions collectives. C'est un système «construit» dans lequel se met en place une régulation conjointe entre les acteurs pour définir les règles de fonctionnements internes (explicites et implicites) et les usages. Cette conception est particulièrement intéressante pour appréhender le changement technologique, de sa conception à ses conséquences sur l'organisation. La technologie est perçue comme structurante dans la mesure où elle détermine des usages. Elle propose ainsi une vision plus large qui enveloppe sous ce concept non seulement la dimension technique mais également les aspects relatifs aux tâches à accomplir, aux processus de transformation et aux connaissances et savoir-faire. Cette approche met particulièrement l'accent sur la dimension humaine, sur les actions et cognitions des individus et groupes d'individus qui utilisent les dispositifs technologiques.

\footnotetext{
${ }^{3}$ Lors de nos entretiens avec un responsable de Microsoft France, ce dernier nous indiquait que selon une récente étude interne, la messagerie électronique constitue le premier outil de travail pour $65 \%$ des salariés dans le secteur tertiaire.

${ }^{4}$ Une étude souligne que près de $96 \%$ des chefs d'entreprise considèrent le courrier électronique comme un outil de simplification de l'organisation du travail dans leur entreprise (Enquête Novamétrie, Microsoft, Cigref, 2006).
} 
Les utilisateurs sont des acteurs qui, au travers du processus d'appropriation, vont faire évoluer à partir de leurs usages le courrier électronique sur des fonctions qui peuvent ne pas être prévues ou pensées par les concepteurs. En interprétant leur environnement et la technologie, les individus construisent la réalité de leur relation avec la technologie. L'une des difficultés de l'analyse de la technologie est qu'elle repose sur les usages. Or, cette notion est complexe car elle n'est pas un objet naturel mais un construit social (Chambat, 1994) qui renvoie à un continuum, allant de la simple adoption à l'utilisation puis à l'appropriation (Breton et Proulx, 2002). En conséquence, la relation de l'usager à la machine est dynamique et se modifie dans le temps, ce qui peut également expliquer que le comportement des usagers paraisse en décalage par rapport à l'emploi préconisé au départ. Par ailleurs, le courrier électronique peut être utilisé pour différentes tâches dans les organisations (communication d'information, échange de documents, discussions asynchrones sur un sujet avec plusieurs acteurs...) et conduire les acteurs à faire évoluer leur utilisation de ce medium.

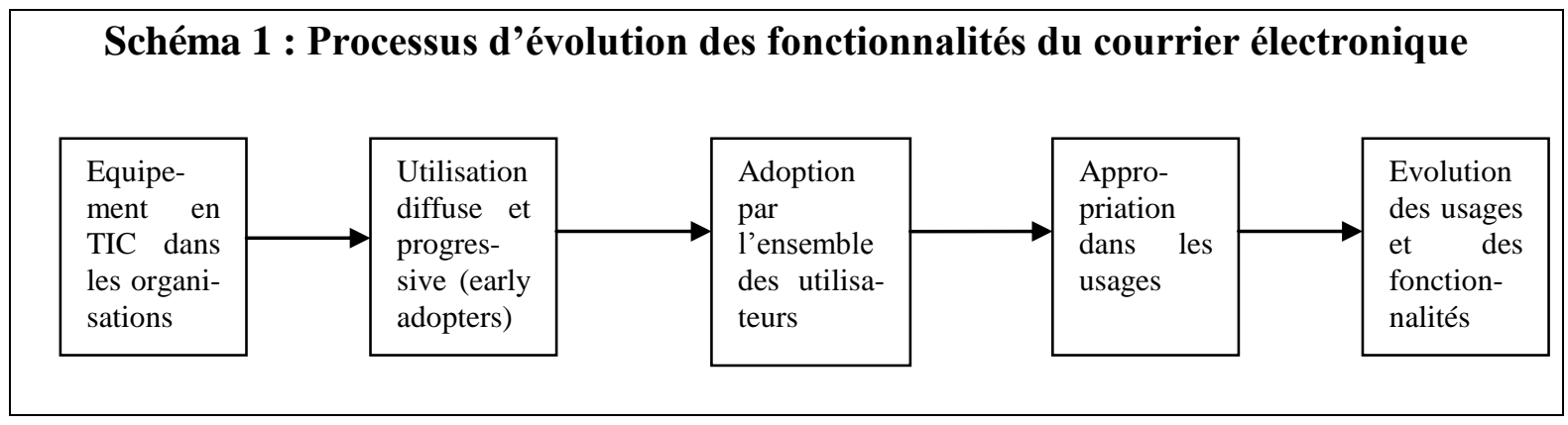

Cette dimension temporelle est importante pour comprendre l'appropriation d'une technologie. Les études qui se sont intéressées à la nature de l'utilisation du courrier électronique mettent en évidence un enrichissement et une diversification de l'usage de l'outil au fur et à mesure du temps (Ducheneaut et Belloti, 2001, 2003 ; McMannus et al., 2002) mais cela reste flou dans la littérature. En utilisant la technologie, les individus redéfinissent les structures, les pratiques mais également la technologie : la technologie est énactée par les acteurs (Orlikowski, 2000). Même si au départ le courrier électronique était considéré comme un média pauvre, notamment dans la théorie de la richesse des médias (Daft et Lengel, 1986 ; Trevino, Daft et Lengel, 1987), certains travaux tels que la théorie de l'expansion du canal (Carlson et Zmud, 1999) montrent que plus on développe des connaissances expérientielles, plus on perçoit la richesse d'un média. Cela peut positionner le courrier électronique comme un média plus riche avec un effet de substitution avec d'autres media de communication. Le contexte d'utilisation et la nature des tâches sont également des éléments pouvant expliquer le choix d'un média de communication par rapport à un autre comme le souligne la théorie du millefeuille (Kalika et al., 2007).

Le courrier électronique possède donc des propriétés émergentes et construites (Fulk et al., 1990 ; Orlikowski, 1992 ; 2000 ; Desanctis et Poole, 1994 ; Yates et al., 1999) qui restent à déterminer car elles sont finalement peu explicitées dans la littérature. C'est ce que nous chercherons à analyser dans notre partie empirique. Dans le prolongement de cette logique, les travaux de Von Hippel (1986) ont montré que les usagers peuvent réinventer une innovation lors de son implémentation. Nous chercherons ainsi à voir quels sont les usages du courrier électronique dans les entreprises à partir d'une approche qualitative étant donné 
notre problématique encore exploratoire sur le thème et que notre objet de recherche n'est pas encore stabilisé du fait des innovations incrémentales successives depuis quelques années (augmentation de la bande passante, ergonomie des logiciels de messagerie, accroissement de l'espace de stockage...).

\section{Les évolutions du courrier électronique vers de nouvelles fonctionna- lités : du médium de communication à l'outil support des applications métiers}

Notre problématique de recherche sur l'évolution des usages et des fonctionnalités des TIC, bien que relativement étudiée dans la littérature, demeure très largement exploratoire compte tenu des résultats très hétérogènes mis en avant dans les différents travaux. Elle se focalise sur un objet technique évolutif (la messagerie électronique) s'inscrivant dans une multitude de contextes très différents les uns des autres, avec de surcroît des facteurs de contingence difficiles à identifier et surtout à mesurer (taille, environnement, maturité de la structure, caractéristiques personnelles des utilisateurs...). Par ailleurs, la nature des tâches et les caractéristiques des utilisateurs (âge, sexe, profession...) constituent des variables pouvant modifier les usages du courrier électronique. Nous allons donc devoir nous restreindre à un cadre particulier dans le cadre de notre recherche.

\subsection{Le courrier électronique : des usages nouveaux à de nouvelles fonctionnalités ?}

Nous avons choisi de développer une stratégie de recherche basée exclusivement sur une approche qualitative au travers d'entretiens de responsables de grandes entreprises et de fournisseurs de solutions logicielles (Miles et Huberman, 1991 ; Yin, 1994). Le choix des grandes entreprises s'expliquent par le fait que nous pouvions ainsi nous assurer que le courrier électronique était un outil largement diffusé parmi l'ensemble des salariés. Parmi les différentes typologies d'utilisateurs dans les entreprises, les études montrent que les managers, à savoir des individus disposant de responsabilité et d'une certaine autonomie, sont des utilisateurs importants du courrier électronique (Markus, 1994 ; Lemarié, 2001) et ils peuvent passer plusieurs heures par jour à répondre et à envoyer des emails (McKeen et Smith, 2004). Ces éléments nous conduisent ainsi à nous focaliser sur cette catégorie d'utilisateurs dans les organisations et à leurs usages du courrier électronique. Le questionnaire concernait donc uniquement cette catégorie d'utilisateurs parmi la population de l'entreprise.

Dans le cadre de notre recherche exploratoire, deux principales sources de données ont été ainsi mobilisées parmi lesquelles dix entretiens semi-directifs d'une durée approximative d'une heure auprès de différentes personnes et une importante collecte de données secondaires auprès de cabinets spécialisés ou de divers organismes d'études sur les SI et les TIC (Novamétrie, l'Idate, IREPP, EBG, CIGREF... ${ }^{5}$ ). L'analyse des données secondaires sou-

\footnotetext{
${ }^{5}$ Institut de Recherches et Prospectives Postales, Electronic Business Group, Club Informatique des Grandes Entreprises Françaises.
} 
ligne également l'importance du questionnement entre les TIC dans les organisations. En ce qui concerne les données primaires, nous avons systématiquement cherché à réaliser des entretiens avec des personnes possédant une vision globale et systémique de l'entreprise, du SI et de l'utilisation du courrier électronique, voire de personnes ressources dans l'implantation de TIC. Les personnes ont été contactées après une recherche sur les sites spécialisés (le Journal du Net, 01 Informatique, les blogs sur les TIC...) et les communautés ou associations s'intéressant aux TIC du point de vue des usages (FING, EBG, CIGREF...). Les questions pour les entretiens ont été élaborées à partir de notre revue de littérature présentée dans la première partie. Les questions ont été posées de la même manière à chaque interlocuteur dans le cadre d'un rendez-vous d'une heure avec une prise de note. Les questions concernaient l'utilisation du courrier électronique dans les entreprises des répondants et chez les clients lorsqu'il s'agissait de prestataires.

Tableau 1 : Liste des questions posées aux répondants

\begin{tabular}{|l|l|}
\hline & \multicolumn{1}{|c|}{ Questions } \\
\hline 1 & Que pensez vous de la diffusion du courrier électronique dans les entreprises ? \\
\hline 2 & $\begin{array}{l}\text { Quelle est la place du courrier électronique dans le portefeuille des outils de } \\
\text { communication des managers ? }\end{array}$ \\
\hline 3 & $\begin{array}{l}\text { Quelles évolutions constatez vous dans l'utilisation du courrier électronique chez } \\
\text { les managers ? }\end{array}$ \\
\hline 4 & $\begin{array}{l}\text { Quelles sont les évolutions du courrier électronique en termes de fonctionnalités } \\
\text { depuis sa création ? }\end{array}$ \\
\hline 5 & $\begin{array}{l}\text { Comment voyez vous évoluer le courrier électronique à l'avenir dans les entre- } \\
\text { prises ? }\end{array}$ \\
\hline
\end{tabular}

Même si cela peut engendrer un biais méthodologique, les personnes interrogées possèdent une vision plus systémique et globale de l'utilisation du courrier électronique qui se positionne dans un portefeuille plus global d'outils de communication. Afin de mieux comprendre les nouvelles fonctionnalités du courrier électronique, nous avons également pris en compte le point de vue des éditeurs de solutions logicielles généralistes et spécialisés en TIC et plus spécialement en solutions de communication et de partage de l'information dans les organisations. La prise en compte des éditeurs nous paraissait importante car leur rôle est déterminant dans une vision élargie de la diffusion des TIC (communication, effet de mode, argumentaire commercial...). Nous avons ensuite procédé à un croisement des données des éléments recueillis durant les entretiens afin de faire ressortir les principaux points concernant les usages et les fonctionnalités du courrier électronique, ainsi que son articulation avec les autres TIC. L'accent a donc été mis entre les différences et les similitudes (Eisendhart, 1989), ainsi que sur les facteurs explicatifs de l'évolution des usages et des fonctions du courrier électronique. 
Tableau 2 : Liste des personnes interrogées

\begin{tabular}{|c|c|c|c|}
\hline \multicolumn{4}{|c|}{$\begin{array}{c}\begin{array}{c}\text { Responsables interrogés sur les pratiques des managers de leur entreprise en matière d'échange } \\
\text { d'informations }\end{array}\end{array}$} \\
\hline Entreprise & Secteur d'activité & Prénom & Fonction \\
\hline $\begin{array}{l}\text { Benchmark } \\
\text { Group }\end{array}$ & $\begin{array}{l}\text { Prestataire de service et presse en } \\
\text { ligne spécialisée }\end{array}$ & Pierre & Directeur associé \\
\hline Groupama & Assurance et produits financiers & Xavier & $\begin{array}{l}\text { DSI Intranet et animateur du } \\
\text { blog «Managers } 2.0 »\end{array}$ \\
\hline ITS Group & SSII & Emilie & $\begin{array}{l}\text { Consultante et animatrice du } \\
\text { blog «savoir en réseaux» }\end{array}$ \\
\hline TDF & Télécommunications & Jean-Luc & DSI \\
\hline Vox Mobilis & $\begin{array}{l}\text { Editeur de solutions logicielles pour } \\
\text { les opérateurs de téléphonie }\end{array}$ & Antoine & $\begin{array}{l}\text { Ingénieur qualité et dévelop- } \\
\text { peur d'une plateforme wiki in- } \\
\text { terne }\end{array}$ \\
\hline \multicolumn{4}{|c|}{ Editeurs de solutions de communication et de messagerie électronique } \\
\hline Akio Software & $\begin{array}{l}\text { Editeur de solution logicielle de ges- } \\
\text { tion multicanal autour de l'email }\end{array}$ & François & Directeur commercial \\
\hline Microsoft & $\begin{array}{l}\text { Editeur de solutions logicielles et bu- } \\
\text { reautiques }\end{array}$ & Gwénael & $\begin{array}{l}\text { Chef de produit unified com- } \\
\text { munications }\end{array}$ \\
\hline Microsoft & $\begin{array}{l}\text { Editeur de solutions logicielles et bu- } \\
\text { reautiques }\end{array}$ & Patrick & Chef de produit Exchange \\
\hline Sight'up & $\begin{array}{l}\text { Editeur de solution logicielle autour } \\
\text { de l'email }\end{array}$ & Franck & PDG \\
\hline Xwiki & Editeur de solution web 2.0 & Thomas & Chef de projet \\
\hline
\end{tabular}

Ces entretiens ont tous été intégralement retranscrits. Ils ont été analysés et interprétés par l'intermédiaire d'une analyse de contenu thématique telle qu'elle est exposée par Bardin (1998). Les textes retranscrits ont été analysés et croisés entre eux à partir de la revue de littérature, ce qui a fait ressortir des conclusions que nous avons mises sous forme de matrices (exemple en annexe). Nous avons posé systématiquement les mêmes questions pour croiser les points de vue de chacun des acteurs et faire ressortir les points saillants, notamment en matière d'évolution du courrier électronique vers de nouvelles fonctionnalités qui expliquent en partie l'effet millefeuille et les problèmes d'articulation avec d'autres TIC ou SI censés être complémentaires. Nous avons choisi d'illustrer nos trois principaux résultats par des encadrés dans la partie suivante. Nos conclusions sont donc le fruit d'itérations successives entre la théorie et nos données empiriques, conformément aux préconisations de Miles et Huberman (2003).

\subsection{Synthèse des résultats : 3 fonctionnalités pour le courrier électronique en com- plément de l'échange d'information}

Le croisement des entretiens suggère une évolution du courrier électronique vers trois fonctionnalités qui tiennent au départ à certains usages détournés de l'outil dans les entreprises suite à l'appropriation du courrier électronique par les managers et à des innovations in- 
crémentales technologiques successives qui vont conférer au courrier électronique des propriétés proches d'autres mediums ou d'autres TIC. Cela permet d'éclairer la littérature sur les propriétés émergentes du courrier électronique. Ces trois fonctionnalités ont pour conséquence de donner une place nodale au courrier électronique dans le portefeuille d'outils de communication dépassant le simple cadre de l'échange d'informations en mode asynchrone comme nous l'avons défini en introduction : une fonction de communication de plus en plus synchrone, une fonction de coordination des tâches et des projets et enfin une fonction de mémorisation organisationnelle.

La première évolution concerne donc la fonction même de communication de messages entre les individus et a déjà été soulignée dans certains travaux. A sa création, le courrier électronique était défini comme un medium de communication asynchrone mais l'accroissement des débits des réseaux en termes de bande passante et la baisse des coûts d'abonnement entraînent une utilisation de plus en plus synchrone au niveau du poste des utilisateurs $^{6}$. A mesure, le mode communication s'est donc transformé et «l'email est de plus en plus utilisé comme un outil synchrone »(Gwénaël, Microsoft), phénomène amplifié par une utilisation possible en situation de mobilité étendue avec l'apparition des outils tels que les Smartphones et Blackberrys. Panteli (2002) montre ainsi que le courrier électronique est plus riche que ne le reflète son classement suivant la théorie de la richesse des médias puisqu'il possède à la fois les propriétés de la communication écrite et orale. En conséquence, le courrier électronique peut se substituer à d'autres outils présents dans l'entreprise ou d'autres modes de communication plus synchrones comme le téléphone notamment («substitution partielle entre l'email et le téléphone qui est réservé à l'hyperurgent » (Pierre, Benchmark Group)). On constate donc que l'utilisation du courrier électronique en mode synchrone amène une réduction du délai d'échange entre les acteurs et parallèlement un prolongement des échanges au-delà des stricts horaires de travail, ce qui peut poser des difficultés de management ${ }^{7}$ (augmentation du stress, dépendance à une connectivité en continu, polychronie...). Le paramétrage des logiciels de messagerie et les habitudes comportementales réduisent le nombre d'informations flottantes (Reix, 2002) mais ne sont pas sans causer des problèmes de management. Cela s'explique aussi par le fait que les entreprises proposent des formations techniques à leurs salariés, notamment pour des systèmes unifiés, mais en général ces dernières n'intègrent pas suffisamment la dimension managériale ou organisationnelle d'après nos entretiens.

\section{Encadré 1 : L'utilisation de l'email par un cadre dirigeant dans une PME}

La PME compte environ 180 personnes avec 3 structures dont 2 en province et elle est spécia-
lisée dans les journaux électroniques et la création de services d'hébergement de sites Internet.
Notre entretien avec l'un des directeurs associés souligne qu'il considère l'email comme son
outil principal de travail. Ce dernier a une vision très positive de l'email (accélération de
l'interaction entre les acteurs et du mode de communication) malgré une réelle conscience des
inconvénients au niveau des usages et de la sécurité des données.

\footnotetext{
${ }^{6}$ On peut ainsi s'apercevoir que des collaborateurs très proches géographiquement (voire même au sein d'un même bureau) s'échangent des emails de manière continue et régulière dans un espace de temps très court (emails « ping-pong »).

${ }^{7}$ A titre d'exemple, on nous a précisé lors de nos entretiens que les managers chez Microsoft ne doivent pas envoyer d'emails après $23 \mathrm{H} 00$ à leurs collaborateurs et chez Bouygues le message envoyé par email ne doit pas avoir plus de 3 destinataires.
} 
D'un point de vue organisationnel en interne, l'email s'est substitué en partie au téléphone, qui est devenu un moyen de communication réservé à «l'hyper-urgent », et la fréquence des réunions s'est également réduite. L'entreprise a mis en place des procédures de travail sur l'intranet à l'aide de tutoriels et elle a créé également une charte des bonnes pratiques axée sur deux points : l'utilisation du poste de travail et les règles de bienséance quant à l'utilisation de l'email en interne et en externe.

Entretien semi-directif réalisé avec le directeur associé de la PME

La seconde évolution du courrier électronique est son utilisation non plus en tant qu'outil de communication mais en tant qu'outil de coordination au niveau intra et inter organisationnel. Certains travaux s'accordent généralement à présenter les systèmes et outils d'information déployés dans les relations interentreprises comme des facilitateurs de la coordination entre les acteurs (Amabile et Gadille, 2006) afin de permettre d'améliorer la productivité (Rice and Blair, 1984). Par ailleurs, la pression des marchés exige une très grande réactivité et nécessite une coordination accrue de personnes, d'équipes, ou d'unités, opérant dans des lieux toujours plus distants (Saunders, 2000) ${ }^{8}$. Le courrier électronique pourrait aussi générer des effets positifs quant à amélioration dans les tâches de communication et de prise de décision (McManus et al., 2002). Cette modularisation des projets engendre alors le besoin d'outils permettant de travailler à plusieurs de manière séquentielle et/ou simultanée indépendamment de l'unité de lieu. Or, il s'avère que le courrier électronique devient dans certains cas un outil collaboratif au détriment d'outils spécifiques tels que les groupwares car un grand nombre d'acteurs en sont équipés et peuvent y avoir accès quel que soit le lieu, voire même de plus en plus en situation de mobilité. On assiste alors à « une compétition entre le travail collaboratif et le courrier électronique » (Xavier, Groupama). Les caractéristiques techniques et les innovations incrémentales (capacité de stockage, pièces jointes, accessibilité en points multiples...) ont conféré au courrier électronique des propriétés propres à certains outils collaboratifs.

Cette substitution partielle aux outils collaboratifs perdure et constitue même dans certaines organisations une routine organisationnelle, au point que même des outils simples de type web 2.0 comme les plateformes wikis ont des difficultés à se mettre en place. L'appropriation rapide du courrier électronique a créé des coûts de changement qui sont difficiles à supprimer lors de l'implémentation de technologies connexes. Certains utilisateurs et développeurs de solutions wiki nous confirmaient ainsi cette difficulté : «l'email reste la voie principale d'échanges d'information entre les salariés » (Antoine, Vox Mobilis) ou «le mail est utilisé pour partager de l'information et le problème est de s'en détacher. Le mail doit servir juste à notifier l'information » (Thomas, Xwiki). Le courrier électronique est donc devenu une véritable porte d'entrée dans les technologies au point que certains éditeurs le considèrent même jusqu'à être un portail d'information pour le CRM ou les ERP. L'un des éditeurs de solutions de messagerie électronique (Sight'Up) nous confirmait d'ailleurs que sa solution pouvait s'interfacer avec des outils de CRM.

\footnotetext{
${ }^{8}$ La société RIM propose avec sa solution Blackberry Enterprise Server une convergence entre la voix, les emails et les applications informatiques de l'entreprise. La solution permet aussi un accès aux autres données que les mails présentent sur l'ordinateur de l'utilisateur.
} 


\section{Encadré 2 : le point de vue d'un éditeur de solutions logicielles (Microsoft)}

Le chef de produit unified communications de Microsoft France souligne que la tendance globale du marché est d'intégrer l'email avec les autres outils de communication et de travail de l'entreprise, avec un réel souci des entreprises de faire en sorte que l'accès et l'utilisation restent simples. L'email devient aussi parallèlement un outil de plus en plus synchrone car il permet le partage d'applications en temps réel. Dans certains cas, certaines solutions centrées sur le courrier électronique (exemple de Business Contact Manager) peuvent satisfaire les besoins des PME et leur éviter de faire l'acquisition d'outils de CRM.

L'interviewé constate également que cette évolution est liée à la démocratisation des outils partagés dans la sphère privée et une demande de transfert de ces outils par les utilisateurs dans les entreprises. On peut distinguer ainsi dans les entreprises des «power users » qui sont des utilisateurs early adopters de nouvelles technologies.

Entretien semi-directif réalisé avec Gwénaël

Le courrier électronique contribue également de plus en plus à donner du sens aux processus au sein des organisations car il va permettre une traçabilité qui nous amène à la dernière fonctionnalité qui est celle de mémorisation et de codification des informations. Dans le prolongement d'une logique de codification des information, cette fonction peut même s'apparenter au concept de mémoire organisationnelle (Walsh et Ungson, 1991; Stein et Zwass, 1995). La connaissance aura besoin d'une information organisée et codifiée pour pouvoir exister étant donné la multiplication des données à traiter dans les organisations, sans compter les changements d'acteurs au sein des entreprises (pyramide des âges, taux de turnover, management des carrières visant à plus de mobilité...). Or, le courrier électronique fait fonction de plus en plus d'outil de sauvegarde des données, notamment grâce à l'augmentation des capacités de stockage ${ }^{9}$ et d'outil de texte et de notes pour des comptesrendus (Xavier, Groupama), ce qui peut brouiller les frontières avec des logiciels de bureautique classique. Toutefois, ce n'est pas sans poser de problème en termes de sécurité des données car il n'existe pas de protection ou de sauvegarde automatique dans les logiciels de messagerie.

L'un de nos interviewés chez Microsoft (Patrick) nous donnait ainsi l'exemple de certains clients chez qui «l'information stratégique est plus dans l'email que dans les ERP à l'heure actuelle » en citant les cadres commerciaux qui rentraient leurs informations dans leur messagerie et non dans les outils de CRM. Par ailleurs, l'un des problèmes réside dans l'archivage des informations et leur localisation, or cela constitue parfois une obligation juridique et comptable pour les entreprises ${ }^{10}$. Les nouvelles fonctionnalités intégrées dans les logiciels de messagerie (archivage, moteur de recherche...) contribuent à positionner le courrier électronique comme un outil de codification des informations et des connaissances. Ces exemples suggèrent ainsi que le courrier électronique constitue une mémoire électronique pour les organisations bien qu'en parallèle on peut souvent constater des outils dédiés à cette fonction de mémorisation des informations (ERP, Intranet, serveurs dédiés...).

\footnotetext{
${ }^{9}$ On peut citer l'exemple de Gspace de Google dans le domaine public dans cette même logique.

${ }^{10}$ Chez Microsoft, l'archivage en local est interdit en raison de la loi Sarban-Oxleys mais un endroit spécifique est prévu pour l'archivage selon l'ancienneté de l'information.
} 


\section{Encadré 3 : l'exemple d'un prestataire de solutions de communication et de partage d'information (Xwiki)}

Xwiki est une start-up qui a été créée en 2004 et qui est spécialisée dans la conception et
l'implémentation de plateformes wiki dont le coût peut varier entre 4000 et $25000 €$.
L'entreprise est un éditeur de solutions logicielles axées sur le service : la plateforme est en
open source et peut s'interfacer avec des applications de groupware comme Lotus Notes qui
comprend par ailleurs une fonction de messagerie. Un lien est donc possible entre le courrier
électronique et les plateformes wiki. Dans la phase de commercialisation, l'entreprise met en
avant l'amélioration du travail collaboratif, de partage et de stockage des informations.
Dans la phase d'implémentation, le courrier électronique est cité comme l'un des principaux
freins évoqués par les développeurs. En effet, les utilisateurs ont beaucoup de difficultés à se
détacher du courrier électronique qui doit servir à notifier l'information plutôt que pour la par-
tager lorsqu'il existe une plateforme wiki.
Entretien semi-directif réalisé avec Thomas et Guillaume

\section{Conclusion}

Les évolutions des métiers et le décloisonnement des organisations amènent de plus en plus les salariés à être multiéquipés et à gérer un portefeuille d'outils de communication. Les individus utilisent souvent plusieurs outils qui sont censés être complémentaires. On retrouve dans plusieurs travaux un principe de substitution ou de complémentarité entre les technologies de la communication (Rowe et Struck, 1999). Le courrier électronique peut facilement s'intégrer avec d'autres technologies et la question n'est plus tant de savoir quelle technologie utiliser mais plutôt comment coordonner de manière efficace les différentes technologies par rapport aux besoins de l'organisation. Pour autant, dans les récents travaux, la diffusion du courrier électronique dans le portefeuille d'outils de communication des managers n'a pas forcément conduit à une réorganisation efficiente de celui-ci, mais plutôt à une augmentation des canaux de communication de l'entreprise qualifiée «d'effet millefeuille» (Boukef et Kalika, 2006 ; Kalika et al., 2007).

Du point de vue des éditeurs de solutions logicielles, il serait opportun de mener une profonde réflexion sur le courrier électronique et ses fonctionnalités. Longtemps pensé et perçu comme un outil de communication asynchrone entre plusieurs acteurs, les développements récents en termes d'appropriation et d'usages doivent amener les éditeurs à repenser l'outil au travers de deux logiques. La première est le déplacement d'une concurrence sur les dominant design ${ }^{11}$ (Abernathy et Utterback, 1978 ; Utterback, 1994) à une concurrence sur l'identité même de l'objet (évolution vers une fonctionnalité de sauvegarde et de gestion des données, outil de text mining, de codification des connaissances...), le tout étant intégré dans une logique systémique avec des éditeurs spécialisés sur des domaines con-

\footnotetext{
${ }^{11}$ Il existe plusieurs définitions des dominant design mais en synthèse cela correspond à une représentation partagée par l'ensemble des acteurs en ce qui concerne les architectures et les valeurs portées par le produit, et qui fonde la compétition sur un petit nombre de fonctions considérées comme essentielles par les clients.
} 
nexes ${ }^{12}$. Cela est également la conséquence de l'évolution des applications métiers qui s'appuient de plus en plus sur la messagerie, l'email devenant alors par exemple un portail d'information pour des applications de CRM ou des ERP comme nous avons pu le voir. La seconde, que l'on pourrait qualifier de learning from using, est une meilleure intégration des besoins souhaités ou développés par les lead users (Von Hippel, 1986), ces derniers s'étant appropriés l'outil au travers d'usages non forcément prévus initialement par les concepteurs. L'engagement des utilisateurs dans le processus de développement de l'application est un facteur de succès. La messagerie électronique pourrait ainsi devenir un artéfact composé de plusieurs modules, l'éditeur définissant les principales règles en tant qu'architecte et les utilisateurs rassemblés au sein de plusieurs communautés développant les sous-modules axés sur des fonctionnalités particulières. Dans le prolongement d'une logique de type 2.0, on pourrait alors avoir une évolution continuelle des fonctionnalités du courrier électronique. Le courrier électronique pourrait évoluer, tant dans ses possibilités de configuration par rapport aux besoins de chaque utilisateur, de son accessibilité déjà très grande mais aussi de son interopérabilité avec des applications métiers propres aux entreprises.

L'un des apports de cette contribution est de souligner qu'il est nécessaire de mieux prendre en compte pour les entreprises le nouveau rôle qu'occupe le courrier électronique dans le circuit de communication entre les acteurs, tant sur le plan de la richesse des communications, de ses possibilités d'évolution et d'interfaçage avec les autres outils électroniques présents dans l'entreprise, et enfin de ses nouvelles fonctionnalités liées de plus en plus aux applications métiers. Le courrier électronique doit être ainsi intégré comme un outil à part entière dans l'architecture globale du SI de l'organisation et, étant donné son niveau d'utilisation par les individus, devenir le point de départ de la diffusion des autres TIC dans le portefeuille des managers. Pour autant, l'évolution du courrier électronique vers les fonctionnalités identifiées mériterait une analyse plus approfondie de type confirmatoire à partir d'une étude quantitative auprès des managers dans les organisations.

\footnotetext{
${ }^{12}$ Un des fournisseurs de solutions logicielles rencontrés (Sight'Up) a développé son système de gestion des messages entrants et sortants en utilisant l'intelligence artificielle.
} 
Exemple de matrice issue des verbatims des répondants sur la fonction de communication du courrier électronique

\begin{tabular}{|l|l|l|}
\hline \multicolumn{1}{|c|}{ Entreprise } & \multicolumn{1}{|c|}{ Répondant } & \multicolumn{1}{c|}{ Extrait du verbatim } \\
\hline $\begin{array}{l}\text { Akio Soft- } \\
\text { ware }\end{array}$ & François & $\begin{array}{l}\text { "Substitution de l'émail au courrier et au fax mais pas } \\
\text { pour le téléphone. Les deux vont de pair mais effecti- } \\
\text { vement nos clients sont plus sensibles aux emails et } \\
\text { semblent parfois privilégier ce moyen de communica- } \\
\text { tion." }\end{array}$ \\
\hline $\begin{array}{l}\text { Benchmark } \\
\text { group }\end{array}$ & Pierre & $\begin{array}{l}\text { "Substitution partielle entre l'email et le téléphone qui } \\
\text { est réservé à l'hyper-urgent" }\end{array}$ \\
\hline Microsoft & Gwenaël & $\begin{array}{l}\text { "L'email est de plus en plus un outil synchrone" } \\
\text { "L'email est utilisé partout, n'importe où et pour n'im- } \\
\text { porte quoi" } \\
\text { "Le partage d'applications en temps réel devient pos- } \\
\text { sible avec l'email" }\end{array}$ \\
\hline TDF & Jean-Luc & $\begin{array}{l}\text { "La messagerie électronique se substitue à la message- } \\
\text { rie vocale du téléphone et de plus en plus au courrier } \\
\text { papier" } \\
\text { "Cela s'explique par le gain de temps, le principe de } \\
\text { multidiffusion du message et le traitement coopératif } \\
\text { en mode texte" }\end{array}$ \\
\hline
\end{tabular}




\section{Bibliographie}

Abernathy W. et Utterback J. (1978), "Patterns of industrial Innovation”, Technology Review, $\mathrm{n}^{\circ} 2$, p. $40-47$.

Amabile S. et Gadille M. (2006), «Coopération interentreprise, système d'information et attention organisationnelle », Revue Française de Gestion, n 164, p. 97-118.

Bailey J. et Pearson S. (1983), " Development of a tool for measuring and analysing computer user satisfaction ", Management Science, Vol. 29, N. 5, p. 530-545.

Bardin L. (1998), «L'analyse de contenu », 9iè édition, Paris, Presses Universitaires de France, 291p.

Boukef N. et Kalika M. (2006), «La théorie du millefeuille, le rôle du contexte », Système d'Information et Management, $\mathrm{n}^{\circ}$ 4, vol 11, p. 29-54.

Breton P. et Proulx S. (2002) «L'explosion de la communication à l'aube du XXIe siècle », La Découverte et Boréal, Paris et Montréal, 400 p.

Carlson J.R. et Zmud R.W. (1999), "Channel expansion theory and the experiental nature of media richeness perceptions", Academy of Management Journal, vol. 42, n², p.153170 .

Chambat P. (1994), « NTIC et représentations des usages », pp. 45-59, in VITALIS, dir., Médias et nouvelles technologies. Pour une socio-politique des usages, Rennes, Éd. Apogée.

Crozier M. et Friedberg E. (1977), L'acteur et le système, Paris, Point Seuil.

Daft R.L. et R.H. Lengel (1986), "Organizational Information Requirements, Media Richness and Structural Design”, Management Science, Vol. 32, n 5, p. 554-571.

Davis F.D. (1989), "Perceived Usefulness, Perceived Ease of Use and User Acceptance of Information Technology", MIS Quarterly, Vol. 13, n³ 3, p. 319-340.

Delone W.H. et McLean E.R. (2003), "The Delone and McLean Model of Information Systems success : A ten year update", Journal of Management Information Systems, 19(4), p. 9-30.

DeSanctis G. et Poole M.S. (1994), « Capturing Complexity in Advanced Technology Use: Adaptive Structuration Theory », Organization Science, Vol.5, n 2, p. 121-146.

Ducheneaut N. et Bellotti V. (2003), «Ceci n'est pas un objet? Talking about objects in Email », Human computer interaction, vol. 18, p. 85-110.

Ducheneaut N. et Bellotti V. (2001), «Email as a habit : An exploration of embedded personal information management», Interactions, vol. 8, n 5 , p. 30-38.

Eisendhart K.M. (1989), "Building theories from case study research", Academy of Management Review, vol 14, n 4, p. 532-550.

Fulk J. Schmitz J. et Steinfield C.W. (1990), « A social influence model of technology use», in Fulk J. et Steinfield C., Organizations and communication technology, Sage publications, p.117-142.

Galbraith, J. (1973), Designing Complex Organizations. Reading, MA: Addison-Wesley Publishing Co.

Kalika M. Boukef N. et Isaac H. (2007), « la théorie du millefeuille et l'usage des TIC dans les entreprises », Revue Française de Gestion, n 172, p. 117-129. 
Karahanna E. et Limayen M. (2000), "E-mail and V-mail usage : Generalizing across technologies", Journal of organizational computing and electronic commerce, vol 10, $\mathrm{n}^{\circ} 1, \mathrm{p}$. 49-66.

Katz J.E., Rice R.E. (2002), Social consequences of internet use: Access, involvement and interaction, Cambridge, MA: MIT Press

Katz M.L. et Shapiro C. (1985), "Network externalities, competition and compatibility", American Economic Review, 75:3, p. 424-440.

Lemarié Y. (2001), «Contribution des nouvelles technologies de l'information à l'exercice des activités managériales » Thèse de doctorat.

Limayem M., Bergeron F. et Richard A. (1997), "Utilisation de la messagerie électroniques mesures objectives versus mesures subjectives", Système d'information et de management, $\mathrm{n}^{\circ} 1$, Vol 2, p. 51-69.

Linlin K. (1996), "Social and non-social uses of electronic messaging systems in organizations", The journal of business communication, Vol 33, n 3, p. 297-326.

McManus D., Sankar C., Carr H., Ford F., (2002) "Intraorganizational versus interorganizational uses and benefits of electronic mail", Information Ressources Management Journal, Vol 15, n³, p.1-13.

Marciniak R. et Rowe F. (1997), «Systèmes d'information, dynamique et organisation », Economica.

Markus M.L. (1987), "Toward a Critical Mass Theory of Interactive Media : Universal Access, Interdependance, and Diffusion", Communication Research, Vol. 14, $\mathrm{n}^{\circ}$ 5, p. 491511.

Markus M.L. (1990), “Toward a 'Critical Mass' Theory of Interactive Media”, dans J. Fulk et C. Steinfield (Eds.), Organizations and Communication Technology, pp.194-218, Sage Publications : London, GB.

Markus M L. (1994), "Electronic Mail as the medium of managerial choice", Organization science, $\mathrm{Vol} 5, \mathrm{n}^{\circ}$ 5, p. 502-527.

McKeen J.D. et Smith H.A (2004), "Electronic communications: Strategies for coping with the deluge", Communications for AIS, vol 13, n¹4, p. 1-19.

McMannus D.J. Sankar C.S. Carr H.H. et Ford F.N. (2002), "Intraorganizational versus interorganizational uses and benefits of electronic mail", Information Resources Management Journal, vol. 15, n³, p.1-13.

Miles M.B. et Huberman A.M. (2003), «Analyse des données qualitatives », De Boeck Université.

Miles M.B. et Huberman A.M. (1991), «Analyse des données quantitatives. Recueil de nouvelles méthodes », De Boeck Université, Bruxelles.

Orlikowski W. (2000), "Using technology and constituting structures: A practice lens for studying technology in organizations", Organization Science, 11: 4, p. 404-428.

Orlikowski W. (1992), "The duality of technology: Rethinking the concept of technology in Organizations ", Organization Science, 3: 3, pp. 398-427.

Panteli N. (2002), « Richness, power cues and email text », Information \& Management, 40 (2), p. 75-86. 
Raymond L. (2002), «L'impact des systèmes d'information sur la performance de l'entreprise », in Faire de la recherche en système d'information, ouvrage coordonnée par F. Rowe, op. cit., Paris, p. 301-320.

Reix R. (1990), «L'impact organisationnel des nouvelles technologies de l'information », Revue Française de Gestion, pp. 100-106.

Reix R. (2002), «Systèmes d'Information et management des organisations », éditions Gestion, Paris.

Rice R.E. et Blair J.H. (1984), "New Organizational Media and Productivity", dans R.E. Rice, et al. (Eds), The New Media : Communication, Research and Technology, Sage : Newbury Park, pp. 184-216.

Rice R.E. et Gatticker U.E. (2001), « New media and organization structuring », in Jablin F.M. et Putman L.L., The new handbook of organizational communication, Sages Publications, pp.544-581.

Rowe F. et Struck D. (1999), "Cultural values, media richness and telecommunication use in an organization", Accounting Management and Information Technologies, vol 9, $\mathrm{n}^{\circ} 3$, pp. 161-192.

Saunders C. (2000), "Virtual teams : Piecing together the puzzle", in Zmud R (ed), Framing the domain of IT management, Cincinnati, Pinnaflex, pp. 29-50.

SESSI. (2006), «TIC et productivité des entreprises », le 4 pages, $\mathrm{n}^{\circ} 223$.

SESSI. (2007), «Tableau de bord des TIC et du commerce électronique », entreprisesménages.

Stein E. et Zwass V. (1995), "Actualizing organizational memory with information technology", Information Systems Research, Vol. 6, No. 2, pp. 85-117.

Trevino L.K. Lengel R.H. et R.L. Daft (1987), "Media Symbolism, Media Richness, and Media Choice in Organizations : A Symbolic Interactionist Perspective", Communication Research, Vol. 14, ${ }^{\circ}$ 5, pp. 553-574.

Utterback J. (1994), "Mastering the dynamics of innovation", Harvard Business School Press, Boston, 253 p.

Van den Hooff B. Groot J. et de Jonge S. (2005), "Situational influences on the use of communication technologies: A meta analysis and exploratory study", Journal of Business Communication, vol 41, $\mathrm{n}^{\circ}$ 2, pp. 4-27.

Von Hippel. (1986), "Lead Users: a source of novel product concepts", Management Science, 32(7): 791.

Walsh J.P. et Ungson G.R. (1991), "Organizational memory", Academy of Management Review, Vol 16, $\mathrm{n}^{\circ} 1$, pp. 57-91.

Weber R. (2004), “The grim reaper: the curse of e-mail”, MIS quarterly, Vol. 28, n³, pp. 3-14.

Yates J. Orlikowski W.J. et Okamura K. (1999), "Explicit and implicit structuring of genres in electronic communication: reinforcement and change of social interaction", Organization science, vol. 10, $\mathrm{n}^{\circ} 1$, pp. 83-103.

Yin R.K. (1994), "Case study research. Design and methods", Sage Publications, London, 1994. 